\title{
Engineering the Calvin-Benson-Bassham cycle and hydrogen utilization pathway of Ralstonia eutropha for improved autotrophic growth and polyhydroxybutyrate production
}

\author{
Zhongkang $\mathrm{Li}^{1,2 \dagger}$, Xiuqing Xin ${ }^{1,2+}$, Bin Xiong ${ }^{1,2}$, Dongdong Zhao ${ }^{1,2}$, Xueli Zhang ${ }^{1,2^{*}}$ and Changhao Bi, ${ }^{1,2^{*}}$ (D)
}

\begin{abstract}
Background: $\mathrm{CO}_{2}$ is fixed by all living organisms with an autotrophic metabolism, among which the Calvin-BensonBassham (CBB) cycle is the most important and widespread carbon fixation pathway. Thus, studying and engineering the $\mathrm{CBB}$ cycle with the associated energy providing pathways to increase the $\mathrm{CO}_{2}$ fixation efficiency of cells is an important subject of biological research with significant application potential.

Results: In this work, the autotrophic microbe Ralstonia eutropha (Cupriavidus necator) was selected as a research platform for CBB cycle optimization engineering. By knocking out either CBB operon genes on the operon or megaplasmid of $R$. eutropha, we found that both CBB operons were active and contributed almost equally to the carbon fixation process. With similar knock-out experiments, we found both soluble and membrane-bound hydrogenases ( $\mathrm{SH}$ and $\mathrm{MBH}$ ), belonging to the energy providing hydrogenase module, were functional during autotrophic growth of R. eutropha. SH played a more significant role. By introducing a heterologous cyanobacterial RuBisCO with the endogenous GroES/EL chaperone system(A quality control systems for proteins consisting of molecular chaperones and proteases, which prevent protein aggregation by either refolding or degrading misfolded proteins) and RbcX(A chaperone in the folding of Rubisco), the culture $\mathrm{OD}_{600}$ of engineered strain increased $89.2 \%$ after $72 \mathrm{~h}$ of autotrophic growth, although the difference was decreased at $96 \mathrm{~h}$, indicating cyanobacterial RuBisCO with a higher activity was functional in R. eutropha and lead to improved growth in comparison to the host specific enzyme. Meanwhile, expression of hydrogenases was optimized by modulating the expression of $\mathrm{MBH}$ and $\mathrm{SH}$, which could further increase the R. eutropha $\mathrm{H} 16$ culture $\mathrm{OD}_{600}$ to $93.4 \%$ at $72 \mathrm{~h}$. Moreover, the autotrophic yield of its major industrially relevant product, polyhydroxybutyrate (PHB), was increased by $99.7 \%$.
\end{abstract}

Conclusions: To our best knowledge, this is the first report of successfully engineering the CBB pathway and hydrogenases of $R$. eutropha for improved activity, and is one of only a few cases where the efficiency of $\mathrm{CO}_{2}$ assimilation pathway was improved. Our work demonstrates that $R$. eutropha is a useful platform for studying and engineering the CBB for applications.

Keywords: Calvin-Benson-Bassham cycle, Carbon fixation pathway, Ralstonia eutropha, Polyhydroxybutyrate

*Correspondence: zhang_xl@tib.cas.cn; bi_ch@tib.cas.cn

${ }^{\dagger}$ Zhongkang Li and Xiuqing Xin contributed equally to this work

${ }^{1}$ Tianjin Institute of Industrial Biotechnology, Chinese Academy of Sciences, Tianjin, China

Full list of author information is available at the end of the article

\section{Background}

The stability and balance of the biosphere is maintained by the flow of carbon back and forth between life forms and the environment through various biogeochemical cycles [1]. According to information from

(c) The Author(s) 2020. This article is licensed under a Creative Commons Attribution 4.0 International License, which permits use, sharing, adaptation, distribution and reproduction in any medium or format, as long as you give appropriate credit to the original author(s) and the source, provide a link to the Creative Commons licence, and indicate if changes were made. The images or other third party material in this article are included in the article's Creative Commons licence, unless indicated otherwise in a credit line to the material. If material is not included in the article's Creative Commons licence and your intended use is not permitted by statutory regulation or exceeds the permitted use, you will need to obtain permission directly from the copyright holder. To view a copy of this licence, visit http://creativeco mmons.org/licenses/by/4.0/. The Creative Commons Public Domain Dedication waiver (http://creativecommons.org/publicdomain/ zero/1.0/) applies to the data made available in this article, unless otherwise stated in a credit line to the data. 
the International Energy Agency, the amount of carbon dioxide released in 2007 was $28.8 \mathrm{Gt}$, which is expected to increase to $40.3 \mathrm{Gt}$ by 2030 and $50 \mathrm{Gt}$ by 2050 , if no appropriate measures are taken [2]. Therefore, we urgently need to develop technologies for carbon recycling or increase the capacity of natural organisms for $\mathrm{CO}_{2}$ fixation.

The Calvin-Benson-Bassham (CBB) cycle, which utilizes the $\mathrm{CO}_{2}$ fixation enzyme ribulose-1,5-bisphosphate carboxylase/oxygenase ( $\mathrm{RuBisCO})$, is a key biological pathway for converting atmospheric $\mathrm{CO} 2$ to organic matter. It is of great significance to the global carbon cycle and crop production, and widely distributed in most autotrophic organisms including plants, algae, cyanobacteria, as well as other photo- and chemoautotrophic bacteria [3]. Apart from the CBB cycle, five other carbon fixation pathways have been discovered in nature, among which the reductive acetyl-CoA pathway has the highest $\mathrm{CO}_{2}$ fixation efficiency under anaerobic conditions, whereby $2 \mathrm{~mol} \mathrm{CO}_{2}$ are fixed into $1 \mathrm{~mol}$ of acetyl-coA using $1 \mathrm{~mol}$ ATP and $4 \mathrm{~mol}$ $\mathrm{NAD}(\mathrm{P}) \mathrm{H}$ [4]. $\mathrm{CBB}$ is more energy intensive, requiring $9 \mathrm{~mol}$ ATP and $6 \mathrm{~mol} \mathrm{NAD}(\mathrm{P}) \mathrm{H}$ for the fixation of $3 \mathrm{~mol} \mathrm{CO}$, but is not sensitive to oxygen and is widely distributed in higher plants, algae and cyanobacteria, which makes improving its efficiency a highly promising prospect [4].

Most research and engineering of the CBB cycle has focused on improving the reaction efficiency of carboxylation by the enzyme RuBisCO, which can be classified into four groups. While all known forms of RuBisCO are composed of catalytic large subunit dimers, the difference lies in different numbers of catalytic larger dimers [5]. It was reported that the assembly of the large dimer requires the synergistic effects of GroES/EL, and assembly of the final RuBisCO octamer comprising the large subunit dimer with RubS requires the RubX chaperone, while the assembly of fully formed RuBisCO does not require the participation of RubX [6,7]. There are a number of strategies to improve the $\mathrm{CO}_{2}$ fixation efficiency, including adaptive evolution of RuBisCO catalytic subunits and the promoter of the CBB operon [8], co-expression of auxiliary pathways, and heterologous introduction of highly catalytic RuBisCO. For example, Lin et al. introduced RuBisCO and RubS from the cyanobacterium Synechococcus elongatus PCC 7942 into tobacco, and achieved an improvement of carbon fixation efficiency. In addition, it was verified that the introduction of a $\mathrm{CO}_{2}$-concentrating mechanism $(\mathrm{CCM})$ could improve the efficiency of $\mathrm{CO}_{2}$ fixation [9]. However, there are very few successful cases, probably due to limited improvement of RuBisCO by adaptive evolution, and the long experimental cycles needed for research in plants.
Ralstonia eutropha H16 (Cupriavidus necator) is a Gram-negative facultatively chemoautotrophic bacterium, which cannot only use fructose, gluconic acid and other organic carbon sources for heterotrophic growth, but also uses $\mathrm{CO}_{2}$ and $\mathrm{H}_{2}$ for autotrophic growth in the presence of $\mathrm{O}_{2}$. Different from most chemoautotrophic bacteria, $R$. eutropha H16 utilizes the CBB cycle for carbon fixation, which is very similar to that of eukaryotes such as plants. Due to its much shorter generation time compared with plants, $R$. eutropha is a potential platform for optimization of the CBB cycle. In addition, this bacterium has been successfully metabolically engineered to produce various chemicals, such as ethanol [10], isobutanol [11], fatty acids, hydrogen [12] and alkanes [13], which suggests that $R$. eutropha $\mathrm{H} 16$ has great potential for development of various biotechnological applications using $\mathrm{CO}_{2}$ sequestration.

With the deciphering of its genome sequence [14, 15], the genes involved in autotrophic growth of $R$. eutropha were identified in silico, including four hydrogenases encoded on the large plasmid, and two copies of the Calvin-Benson-Bassham (CBB cycle) operon, one on chromosome 2 and one on the large plasmid. The CBB operon of $R$. eutropha on chromosome includes 14 genes, which are cbbR, L, S, X, Y, E, F, P, T, Z, G, K, A and B. These genes and their coded enzyme of $\mathrm{CBB}$ operon is listed in Additional file 1: Table S3. The copy on mega plasmid has all genes except cbbB. Hydrogenases catalyze the oxidation of hydrogen to form $2 \mathrm{e}^{-}$and $2[\mathrm{H}]^{+}$. The four hydrogenases of $R$. eutropha $\mathrm{H} 16$ are membrane-bound hydrogenase $(\mathrm{MBH})$, soluble hydrogenase $(\mathrm{SH})$, regulatory hydrogenase $(\mathrm{RH})$ and a fourth NiFe hydrogenase (Hyd4) [16]. All of them are oxygen-resistant members of the [NiFe]-hydrogenase family. The two hydrogenases $\mathrm{MBH}$ and $\mathrm{SH}$, which have distinct functions, have been purified and analyzed [17]. MBH is composed of HoxK and HoxG structural subunits, which are anchored to the membrane by HoxZ. This complex delivers electrons to B-type cytochrome, and further to the electron transport chain to provide energy for $R$. eutropha H16 cells. Protons are delivered to periplasm [18]. SH is heterotetrameric complex composed of HoxH, Y, F, and U subunits, which delivers protons and electrons to $\mathrm{NAD}^{+}$to synthesize NADH for cell growth and biosynthetic reactions. Its maturation requires a series of accessory proteins to assist its complex assembly. The third hydrogenase, $\mathrm{RH}$, is a $\mathrm{H}_{2}$-sensing regulatory [NiFe]-hydrogenase consisting of HoxB, $\mathrm{C}$ and $\mathrm{J}$ subunits. It acts as a signal protein that controls the functional expression of $\mathrm{MBH}$ and $\mathrm{SH}$, independent of intracellular energy and NADH status [17]. Hy4 hydrogenase is one of the least well-characterized hydrogenases. It consists of the two structural subunits PHG064 and PHG065, as well as a number of auxiliary 
proteins, including hypF3, hypC2, hypD2, hypE2, hypA3, and hypB3, whose physiological roles and expression mechanisms are still not fully known [16]. On the other hand, the CBB operon on the R. eutropha chromosome is much better understood. It encodes an inversely arranged lysine family transcriptional regulator protein $C b b R$, and a polycistronic $\mathrm{CBB}$ expression cassette. Moreover, the two copies of the CBB operon have high homology. The lysine family regulatory protein located on the megaplasmid is not complete, but the CbbR expressed from the chromosome still regulates the expression of the $\mathrm{CBB}$ operon on the megaplasmid [19].

Previous studies provided a basis for engineering the autotrophic metabolic pathways of $R$. eutropha. In this study $R$. eutropha H16 was selected for research and engineering due to its potential to be developed as an efficient and convenient platform for optimization of the $\mathrm{CBB}$ cycle, as well as an efficient $\mathrm{CO}_{2}$-sequestrating cell factory for various biotechnological applications.

\section{Results and discussion}

Determination of the contribution of the CBB operons and hydrogenases to autotrophic growth of $R$. eutrophic H16

The CBB cycle is an expensive metabolic pathway that consumes large amounts of energy and reducing equivalents. The production of $1 \mathrm{~mol}$ of 3-PG from $3 \mathrm{~mol}$ of $\mathrm{CO}_{2}$ requires $9 \mathrm{~mol}$ of ATP and $6 \mathrm{~mol}$ of NADPH. In R. eutropha H16 cells, the hydrogenase systems are employed to provide both the energy and the reducing equivalents for the CBB cycle [4]. The hydrogenases of $R$. eutropha $\mathrm{H} 16$ are insensitive to oxygen, which is rather unusual. They catalyze the oxidation of molecular hydrogen into protons and electrons. Electrons are then transferred to membrane-bound or cytoplasmic electron carriers with oxygen as the terminal electron acceptor [20]. This process either generates the proton electromotive force for ATP production or provides NADH. [21], or is used for the regeneration NADH through the cytoplasmic electron transport chain [22]. There are few studies on the three main hydrogenases of the autotrophic system of $R$. eutropha H16, i.e. SH, MBH and $\mathrm{RH}$, and their individual contribution to carbon fixation and autotrophic growth is unknown. It was reported that the CBB operon on chromosome 2 of $R$. eutropha H16 contains $13 \mathrm{CBB}$ coding sequences, along with a $c b b R$ gene on the negative strand, while the CBB operon on the megaplasmid of $R$. eutropha H16 consists of 12 CBB coding sequences with a deficient $c b b R$ gene [19]. The CbbR expressed from chromosome 2 was considered to control the expression of both CBB operons [19]. Here, we estimated which hydrogenase or $\mathrm{CBB}$ operon is more important for autotrophic growth by constructing corresponding knockout strains.

The knockout strains and strains carrying overexpression plasmids were subjected to autotrophic fermentation, which is performed in minimal medium supplemented with only a gas mixture comprising $\mathrm{H}_{2}$, $\mathrm{CO}_{2}$, and $\mathrm{O}_{2}$ at a volume ratio of 7:1:1, but no organic substrate is provided. According to non-systematic experiments, the growth of $R$. eutropha could reach 14 of OD 600 in the autotrophic fermentation at $369 \mathrm{~h}$ (data not shown). Under such conditions, $\mathrm{CO}_{2}$ was the only carbon source for the synthesis of cellular building blocks, and the cell growth efficiency was assumed to be directly correlated with the carbon fixation efficiency. The strains are summarized in Additional file 1: Table S1, and their autotrophic growth phenotype is illustrated in Additional file 1: Figure S1. The results indicated that a double knockout of both $\mathrm{MBH}$ and $\mathrm{SH}$ hydrogenases completely eliminated the autotrophic growth capacity of $R$. eutropha $\mathrm{H6}$, while a single deletion of either $\mathrm{MBH}$ or SH only partly affected the autotrophic growth. Based on the growth profile, the deletion of $\mathrm{SH}$ had a more significant impact (Additional file 1: Figure S1A). Thus, while both hydrogenases were functional during autotrophic growth of $R$. eutropha $\mathrm{H} 6$ and contributed to the cell metabolism, the results indicated that $\mathrm{SH}$ probably played a more significant role.

In the case of the CBB enzymes, both single deletion of $\mathrm{RuC}$ (RuBisCO operon on Chromosome 2) or RuP (RuBisCO operon on the megaplasmid) affected the autotrophic growth efficiency, and the growth decrease was similar for both operons (Additional file 1: Figure $\mathrm{S1B}$ ), which indicated that both $\mathrm{CBB}$ operons were active and contributed almost equally to the carbon fixation process.

To determine if there was a possible polar effect of the knockout experiments, complementation experiments were performed to see if we could restore the autotrophic growth capacity of the double knock out strains of RuBisCO or hydrogenases via plasmid-based expression of the knocked-out genes. The complemented strain $\mathrm{H} 16 \Delta \mathrm{RuP} \Delta \mathrm{RuC}(\mathrm{pRrub})$ carrying plasmid pRrub Additional file 1: Table S1, expressing rubisco units of $R$. eutropha, recovered its autotrophic growth ability, indicating that there was no polar effect of the $\mathrm{RuBisCO}$ knockouts (Additional file 1: Figure S1C). However, the growth of the complemented strain was not as good as the wild type strain, which we considered as an acceptable variation in the gas fermentation process. In addition, the complementation of the two hydrogenases in strain $\mathrm{H} 16 \triangle \mathrm{MBH} \triangle \mathrm{SH}$ was not successful. Considering the difficulties of a manipulating large number of genes in 
$R$. eutropha, the failed complementation experiment does not necessarily indicate a polar effect for the knockout.

In summary, we determined that both the $\mathrm{SH}$ and $\mathrm{MBH}$ hydrogenases contribute to the autotrophic growth of $R$. eutropha with $\mathrm{SH}$ significantly more important than $\mathrm{MBH}$, and both $\mathrm{CBB}$ operons are active in the carbon fixation process.

\section{Engineering the CBB cycle for improved autotrophic growth of $R$. eutropha}

The autotrophic metabolism of $R$. eutropha H16 constitutes of the $\mathrm{CBB}$ cycle and hydrogenases. In the $\mathrm{CBB}$ cycle, RuBisCO catalyzes the carboxylation reaction converting ribulose-1,5-diphosphate and $\mathrm{CO}_{2}$ to generate 2 molecules of 3-phosphoglyceric acid for the synthesis of organic carbon compounds. The efficiency of RuBisCO is low and it is considered the speed-limiting step of the CBB cycle (Fig. 1) [9]. Therefore, we intended to improve the carbon fixation efficiency of $R$. eutropha $\mathrm{H} 16$ by increasing the efficiency of its RuBisCO enzyme. The $\mathrm{RuBisCO}$ with the highest reported efficiency is that from the cyanobacterium Synechococcus sp. PCC 7002 [8]. Due to the complex structure of RuBisCO, its heterologous folding and maturation might not be ideal and require chaperones or accessory proteins. Previous studies have shown that the GroES/EL chaperone system of $E$. coli is of great significance for the folding of heterologous
RuBisCO proteins [23]. Therefore, in this study we attempted to overexpress the endogenous $\mathrm{RuBisCO}$ from $R$. eutropha or the heterologous cyanobacterial $\mathrm{RuBisCO}$, coupled with various chaperone systems to find an optimal strategy for increasing the $\mathrm{CO}_{2}$ fixation capacity.

Overexpression plasmids with different combinations of $\mathrm{RuBisCO}$ genes and chaperone systems were constructed based on the pBBR1-MCS multiple-copy vector as listed in Additional file 1: Table S1. The strains carrying these plasmids were subjected for autotrophic gas fermentation in minimal medium supplemented with the gas mixture as described above. However, we found that most of the engineered strains had decreased growth compared with the control strain carrying an $r f p$ expression plasmid with the same vector backbone (Fig. 2). The strains with decreased growth included those overexpressing only $\mathrm{RuBisCO}$ genes, RuBisCO genes together with the $E$. coli chaperone genes groES/groEL, and the endogenous $R$. eutropha $\mathrm{RuBisCO}$ together with endogenous groES/groEL. Only the strain H16(pRub_cyano, pGroESL_R), which overexpresses the Synechococcus sp. PCC 7002 RuBisCO genes together with the endogenous chaperone genes groES/groEL showed an increased growth phenotype. Its $\mathrm{OD}_{600}$ after $72 \mathrm{~h}$ of growth was $89.2 \%$ higher than that of the control strain. However, when fermentation was processed through $96 \mathrm{~h}$, the difference between

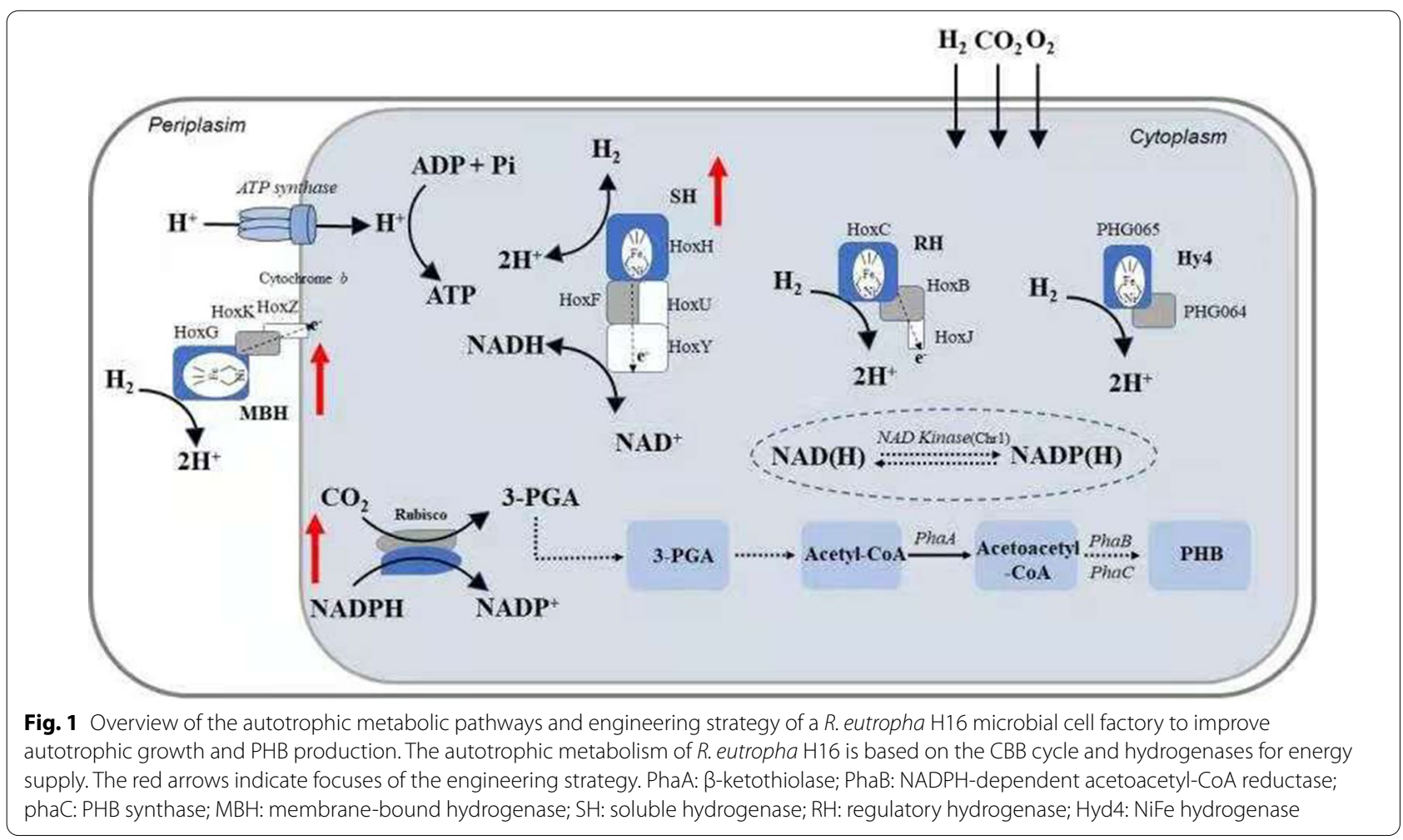



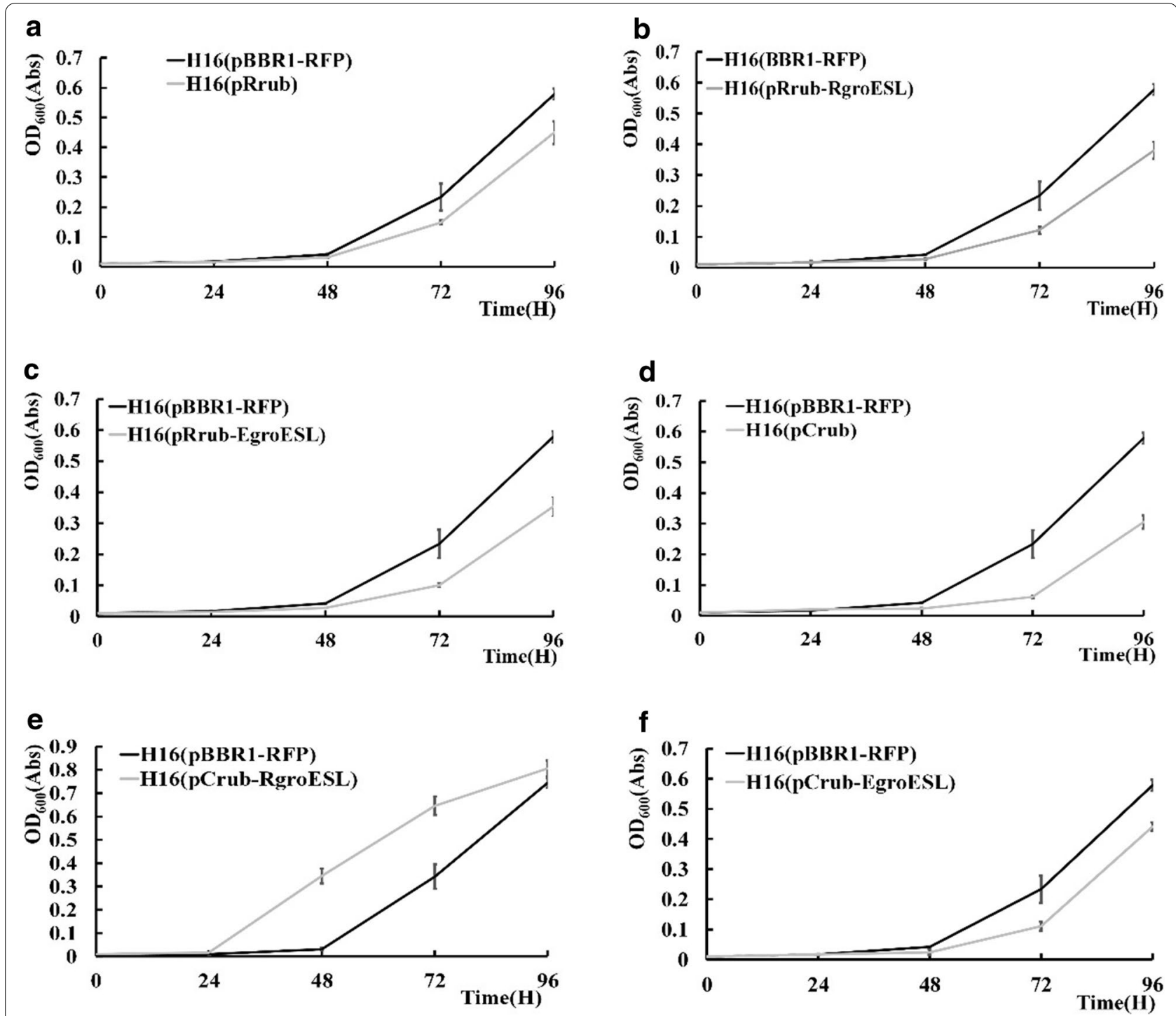

Fig. 2 Optimization of RuBisCO expression in R. eutropha H16. a Overexpression of RubL and RubS subunits from R. eutropha H16; $\mathbf{b}$ Overexpression of RubL, RubS subunits and GroES/GroEL from R. eutropha; c Overexpression of RubL, RubS subunits from R. eutropha and GroES/GroEL subunits from E. coli; d Overexpression of RubL, RubS and RubX from Synechococcus sp. PCC 7002; e Overexpression of RubL, RubS and RubX subunits from Synechococcus and GroES/GroEL subunits from R. eutropha; f Overexpression of RubL, RubS and RubX subunits from Synechococcus and GroES/ GroEL subunits from E. coli; H16(pBBR1-RFP) is the control strain for each experiment. The values and error bars represent the means and SD of triplicate experiments

engineered and control strain was reduced (Fig. 2e). The engineered strain had a shorter lag phase probably due to the un-regulated expression of.

These results indicated that the assembly and maturation of a functional cyanobacterial RuBisCO in $R$. eutropha was successfully achieved with the assistance of overexpressed endogenous GroES/EL chaperons, as well as confirming the feasibility of increasing the carbon fixation efficiency of $R$. eutropha using heterologous RuBisCO enzymes.

\section{Engineering of the hydrogenase module and CBB cycle} for improved autotrophic growth of R. eutropha C5

To engineer the hydrogenase systems, the genes encoding each hydrogenase were overexpressed using the same plasmids that were constructed for complementation in the knockout experiments, and the engineered strains were analyzed for their autotrophic growth phenotype. As illustrated in Fig. 3, while overexpression of the hox$A B C J$ genes encoding the $\mathrm{RH}$ hydrogenase decreased the growth (Fig. 3a), overexpressing the PHG064 and 

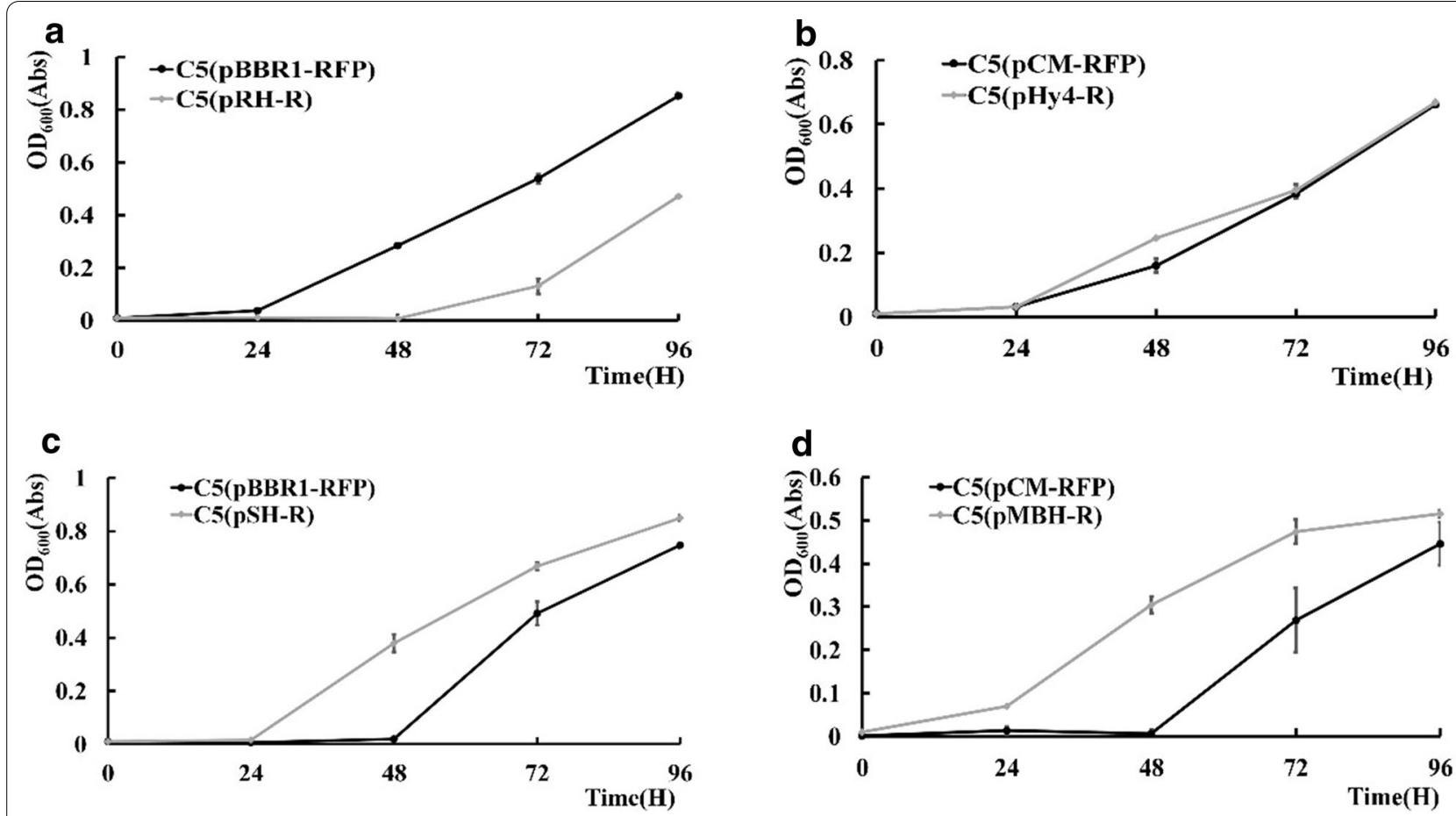

Fig. 3 Autotrophic growth status of R. eutropha C5 strains overexpressing RH, SH, MBH or Hy4 hydrogenase. a RH structure subunits; $\mathbf{b}$ Hy4 subunits; c SH subunits; d MBH subunits; C5(pBBR1-RFP) is the control strain for each experiment. The values and error bars represent the means and SD of triplicate experiments

PHG065 genes encoding Hy4 had no effect. By contrast, expression of either hoxFUYHI encoding $\mathrm{SH}$ or hoxKGZ encoding MBH had a positive effect on the autotrophic growth. Compared with the control, after $96 \mathrm{~h}$ of autotrophic growth, the $\mathrm{OD}_{600}$ of the $\mathrm{SH}$ overexpression strain C5(pRH_R) and MBH overexpression strain C5(pMBH_R) increased 13.8 and 58.7\%, respectively.

Since the plasmid expression system has limitations for more complex engineering, we intended to engineer the hydrogenase systems by modification the R. eutropha genome. The BBaJ_23100,BBa__23109 and BBaJ_23119 promoters from E. coli were selected (Additional file 1: Table S3) to modulate the expression of the $\mathrm{MBH}$ and $\mathrm{SH}$ hydrogenase operons. The corresponding strains were constructed by replacing the original promoters with the $\mathrm{BBa}$ promoters. Because the R. eutropha $\mathrm{C} 5$ strain we constructed is simpler to transform [24, 25], we decided to use it instead of $\mathrm{H} 16$ for these experiments. As shown in Fig. 4a, while the promoters BBaJ_23100 and BBaI_23109 failed to increase the autotrophic growth, BBaJ_23119 with a stronger efficiency was able to increase the growth when inserted instead of the original MBH promoter. This effective regulator was introduced to increase the expression of the $\mathrm{SH}$ operon to obtain the strain C5-sh19. Subsequently, strain C5-shmbh19 in which both the $\mathrm{MBH}$ and $\mathrm{SH}$ operons were upregulated by BBaJ_23119 were constructed. Both strains C5-sh19 and C5-sh-mbh19 were found to have increased autotrophic growth compared with the parent strain, and C5-shmbh19 had a slightly higher growth than C5-sh19.

In this part, we modulated the expression of both $\mathrm{SH}$ and $\mathrm{MBH}$ by both plasmid-based overexpression and chromosomal promoter modulation. The results suggested that increased expression of both hydrogenase operons benefited the autotrophic growth of $R$. eutropha (Fig. 4b).

Engineering of both the $\mathrm{CBB}$ module and the hydrogenase module improved the autotrophic growth and PHB production of $R$. eutropha

The genomic engineering of the hydrogenase operons provided a basis for engineering a strain in which both the $\mathrm{CBB}$ module and the hydrogenase module were engineered. By transforming strain C5-sh-mbh19 with the plasmids pRub_cyano and pGroESL_R, the strain C5-shmbh19(pRub_cyano, pGroESL_R) was obtained. Combining all the findings of this research, this strain may represent the most deeply engineered $R$. eutropha strain to date in terms of its autotrophic metabolism.

Ralstonia eutropha can accumulate polyhydroxybutyrate (PHB) [26], which is a potential bioplastic material of great interest, in intracellular granules. Therefore, 

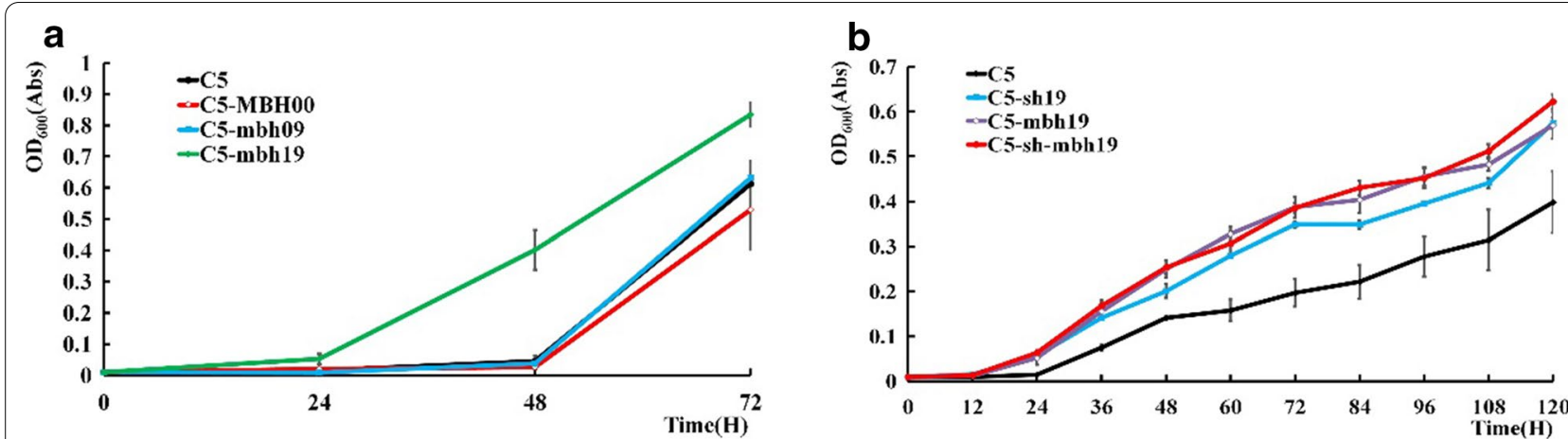

Fig. 4 Autotrophic growth status of R. eutropha C5 strains with chromosomally upregulated expression of SH and MBH hydrogenases. a Promoter modification of the $\mathrm{MBH}$ gene cluster by replacing the native promoter with artificial promoters of different intensity; $\mathbf{b}$ promoter modulation of the MBH gene cluster, the SH gene cluster, or both $\mathrm{SH}$ gene clusters simultaneously. C5 is the control strain for each experiment. The values and error

bars represent the means and SD of triplicate experiments

both the autotrophic growth status and PHB production capacity of C5-sh-mbh19(pRub_cyano, pGroESL_R) were evaluated. As illustrated in Fig. 5, the engineered strain displayed a substantial improvement in both the growth phenotype and PHB production, with increases of $93.4 \%$ and $74.7 \%$ in $96 \mathrm{~h}$ respectively compared to the parent strain. C5-mbh-sh19(pRub_cyano, pGroESL_R) produced $0.34 \mathrm{~g} / \mathrm{g}$ PHB, which was a significant improvement over the $0.17 \mathrm{~g} / \mathrm{g}$ produced by H16(pBAD-RFP).

The reason for selection of $\mathrm{C} 5$ strain as the parent strain in this work is due to the heavy genetic manipulation work requirement for engineering the autotrophic system of $R$. eutropha. The C5 strain provided a more convenient experimental subject for this work, which was constructed in our lab by knocking out only two putative restriction endonuclease genes H16_A0006 and H16_ A0008-9 [25]. Since these two genes are by no means related to any autotrophic process, therefore we consider that $\mathrm{C} 5$ should perform the same with the wild type H16 strain, in terms of the metabolic manipulation towards the autotrophic system.

\section{Conclusions}

The introduction of heterologous $\mathrm{RuBisCO}$ with higher efficiency into $R$. eutropha H16 coupled with overexpression of the endogenous GroES/EL chaperones was able to increase its autotrophic growth efficiency. At the same time, we found that both the $\mathrm{MBH}$ and SH hydrogenases contribute to the energy supply for autotrophic growth. We screened out a strong promoter from E. coli, BBaJ_23119, which could improve the expression of the chromosomal $\mathrm{SH}$ and $\mathrm{MBH}$ hydrogenase operons and increase the autotrophic growth. Finally, modification of both the CCB and hydrogenase modules was combined
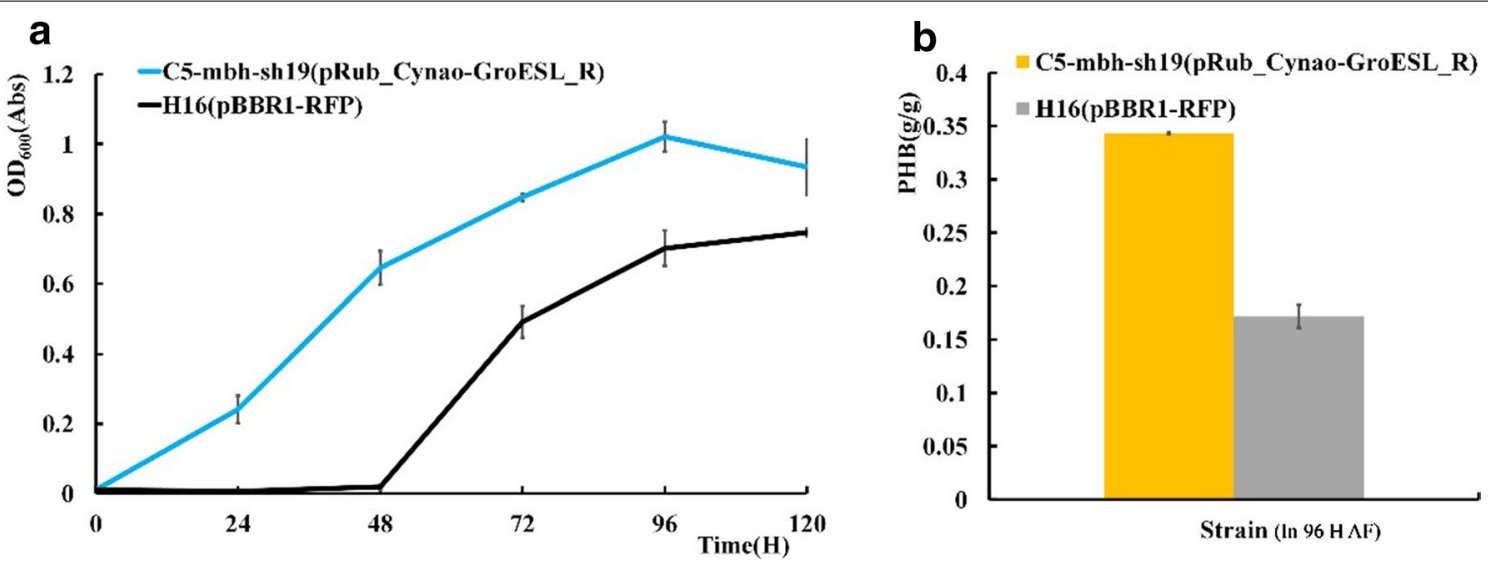

Fig. 5 Growth and PHB production time course of the highly engineered strain C5-sh-mbh19(pRub_cyano, pGroESL_R) and the control strain in minimal medium with gas supplementation. a Growth curve of strain C5-sh-mbh19(pRub_cyano, pGroESL_R) and the control strain. b PHB production of strain C5-sh-mbh19(pRub_cyano, pGroESL_R) and the control strain. In 96 H AF: PHB production strain is in 96 h(H) autotrophic fermentation. The values and error bars represent the means and SD of triplicate experiments 
in one deeply engineered strain. With supplementation of only $\mathrm{H}_{2}, \mathrm{CO}_{2}$, and $\mathrm{O}_{2}$, both the autotrophic growth and PHB production were significantly increased. Although there might be other limiting factors, RuBisCO carboxylation efficiency is one of the key rate limiting reaction that restricts the $\mathrm{CBB}$ cycle and determines the plant carbon fixation efficiency and crop yield [27]. Increasing the efficiency of the RuBisCO system is one of the possible directions for significantly improving crop production. The successful optimization of autotrophic systems in bacteria provides an alternative and probably a better platform for the study and future improvement of carbon assimilation to increase crop yields.

\section{Methods}

\section{Strains and culture conditions}

The strains and plasmids used in this work are listed in Additional file 1: Table S1. E. coli S17 was used as the intermediate host for conjugational transfer. It was cultured at $37^{\circ} \mathrm{C}$ and $250 \mathrm{rpm}$ in lysogeny broth (LB) with appropriate antibiotics. $R$. eutropha $\mathrm{H} 16$ was grown at $30{ }^{\circ} \mathrm{C}$ and $250 \mathrm{rpm}$ in $\mathrm{LB}$ or minimum medium with appropriate antibiotics. Antibiotic concentrations were $10 \mathrm{mg} / \mathrm{L}$ for gentamicin, $200 \mathrm{mg} / \mathrm{L}$ for kanamycin, and $30 \mathrm{mg} / \mathrm{L}$ for chloramphenicol. Plates were prepared by adding $1.5 \%$ agar to the liquid medium. Minimal medium contained $5.226 \mathrm{~g} / \mathrm{L} \mathrm{NaH} \mathrm{PO}_{4} \cdot 2 \mathrm{H}_{2} \mathrm{O}, 11.55 \mathrm{~g} / \mathrm{L}$ $\mathrm{Na}_{2} \mathrm{HPO}_{4} \cdot 12 \mathrm{H}_{2} \mathrm{O}$, and $0.453 \mathrm{~g} / \mathrm{L} \mathrm{K}_{2} \mathrm{SO}_{4}, \mathrm{pH}: 6.8-7.0$.

\section{Plasmid construction}

All plasmids and DNA oligo primes were designed using j5 DeviceEditor or Clonemanager [28]. All plasmids were assembled using Gibson assembly or CPEC [29, 30]. DNA segments were PCR-amplified using PrimeSTAR or Phusion High fidelity DNA polymerase (Takara, Japan). All DNA segments were purified using the SanPrep Column DNA Gel Extraction Kit (Sangon Biotech, China) before assembly. All plasmids were sequenced by Genewiz corporation (China) and all primes used in this study are listed in Additional file 1: Table S2.

Preparation of competent cells and electro-transformation Competent cells of E. coli S17 and R. eutropha $\mathrm{H} 16$ were prepared as described elsewhere [24, 25]. For E. coli transformation, we used pre-chilled sterile 1-mm gap electroporation cuvettes. After electroshock at $1.8 \mathrm{kV}$, $1 \mathrm{~mL} \mathrm{LB}$ was added ant the cell suspension transferred to a $1.5 \mathrm{~mL}$ centrifuge and incubated at $37^{\circ} \mathrm{C}$ and $250 \mathrm{rpm}$ for $1 \mathrm{~h}$ for regeneration, after which the cells were spread on LB agar plates with the appropriate antibiotics. For $R$. eutropha transformation, we used 2-mm gap width cuvettes with an electric pulse of $2.3 \mathrm{kV}$, and regenerated the cells for $2 \mathrm{~h}$ in a $30{ }^{\circ} \mathrm{C}$ incubator.

\section{Gas fermentation}

A batch fermentation system and a continuous fermentation system were used to analyze the cell growth status and PHB synthesis, respectively. The gas mixture used for autotrophic fermentation was composed of $\mathrm{H}_{2}, \mathrm{CO}_{2}$ and $\mathrm{O}_{2}$ at a ratio of 7:1:1. The $\mathrm{H}_{2}$ was supplied by a generator, and the $\mathrm{CO}_{2}$ and $\mathrm{O}_{2}$ were provided from gas tanks. The discontinuous fermentation system was composed of $100 \mathrm{~mL}$ headspace bottles with $10 \mathrm{~mL}$ minimal medium. For batch fermentation, the initial $\mathrm{OD}_{600}$ of $R$. eutropha was set to 0.01 , after which the fermentation bottles were sparged with the gas mixture for $3 \mathrm{~min}$ to fill the head space. During the fermentation, the headspace was sparged with fresh gas mixture every $12 \mathrm{~h}$ and the bottles were incubated at $30^{\circ} \mathrm{C}$. For the continuous fermentation system, a $500 \mathrm{~mL}$ bioreactor (Additional file 1: Figure S1) with $150 \mathrm{~mL}$ of minimal medium was used. The gas mixture was continuously sparged into the fermenter at a flow rate of $60-70 \mathrm{~mL} / \mathrm{min}$. The continuous fermentation was conducted at $30^{\circ} \mathrm{C}$.

\section{Extraction and GC analysis of the PHB monomer 3-hydroxybutyric acid}

After autotrophic fermentation, R. eutropha H16 cells were centrifuged at $8000 \mathrm{rpm}$ for $5 \mathrm{~min}$, washed twice with $\mathrm{ddH}_{2} \mathrm{O}$, and put in a $70{ }^{\circ} \mathrm{C}$ oven for drying. The dry cells were weighed in a screw-capped glass tube, suspended in $2 \mathrm{~mL}$ methanol with $3 \%$ sulfuric acid and $2 \mathrm{~mL}$ chloroform, and heated at $100{ }^{\circ} \mathrm{C}$ for $4 \mathrm{~h}$ to achieve methyl esterification. After the sample was cooled, $1 \mathrm{~mL}$ of distilled water was added and vortexed for $5 \mathrm{~min}$. The lower organic phase after static stratification was used for GC analysis after organic membrane filtration [31].

An Agilent 7890B gas chromatography system (Agilent, USA) equipped with an HP-5 column (Agilent, USA) was used for analysis. The GC program consisted of two stages. The first stage had an initial temperature of $80^{\circ} \mathrm{C}$ and a final temperature of $140{ }^{\circ} \mathrm{C}$. The initial hold time was $1.5 \mathrm{~min}$ and the rate of temperature increase was $30{ }^{\circ} \mathrm{C} / \mathrm{min}$. The second stage had an initial temperature of $140{ }^{\circ} \mathrm{C}$ and a final temperature of $220^{\circ} \mathrm{C}$. The hold time was $4.5 \mathrm{~min}$ and the rate of temperature increase was $40{ }^{\circ} \mathrm{C} / \mathrm{min} . \mathrm{N}_{2}$ was used as carrier gas and 3-hydroxybutyric acid standard (Sigma-Aldrich, USA) was used for quantification of the cellular PHB content. The standard GC curve of PHB is shown in supplemental Fig. 2.

\section{Supplementary information}

The online version contains supplementary material available at https://doi. org/10.1186/s12934-020-01494-y.

Additional file 1. Supplement figures and tables. 


\section{Acknowledgements}

The gene coding Cyanobacterial Rubisco derived from Synechococcus Sp.pcc.7002 was kindly bestowed by professor Yin Li, Institute of Microbiology CAS

\section{Authors' contributions}

LZ planned and performed the experiments, wrote the manuscript, analyzed and interpreted the data. BC and ZX supervised the study, wrote the manuscript, designed the experiments, analyzed and interpreted the results. ZD, XX and XB wrote the manuscript, analyzed and interpreted the data. All authors read and approved the final manuscript.

\section{Funding}

This research was financially supported by the The National Key Research and Development Program of China (2018YFA0901300), Key Research Program of the Chinese Academy of Science (KFZD-SW-215), and National Natural Science Foundation of China (31522002, 31770105)

\section{Availability of data and materials}

We provide supporting and necessary data for publication of the article. All supporting data is present in the article and the supplemental material documents. The strains and plasmids associated with this work will be physically available upon request from the authors.

\section{Ethical approval and consent to participate}

Not applicable.

\section{Consent for publication}

I hereby give the Journal of Microbial Cell Factories the right and permission to publish this article.

\section{Competing interests}

The authors declare that they have no competing interests.

\section{Author details}

${ }^{1}$ Tianjin Institute of Industrial Biotechnology, Chinese Academy of Sciences, Tianjin, China. ${ }^{2}$ Key Laboratory of Systems Microbial Biotechnology, Chinese Academy of Sciences, Tianjin, China.

Received: 11 June 2020 Accepted: 5 December 2020 Published online: 11 December 2020

\section{References}

1. Cox PM, Betts RA, Jones CD, Spall SA, Totterdell IJ. Acceleration of global warming due to carbon-cycle feedbacks in a coupled climate model. Nature. 2000:408:184-7.

2. Quadrelli EA, Centi G, Duplan JL, Perathoner S. Carbon dioxide recycling: emerging large-scale technologies with industrial potential. ChemSusChem. 2011:4:1194-215.

3. Andersson I. Catalysis and regulation in Rubisco. J Exp Bot. 2008:59:1555-68

4. Nanette R. Boyle JAM: computation of metabolic fluxes and efficiencies for biological carbon dioxide fixation. Metab Eng. 2011;13:150-8.

5. Tabita FR, Satagopan S, Hanson TE, Kreel NE, Scott SS. Distinct form I, II, III, and IV Rubisco proteins from the three kingdoms of life provide clues about Rubisco evolution and structure/function relationships.J Exp Bot 2008;59:1515-24.

6. Saschenbrecker S, Bracher A, Rao KV, Rao BV, Hartl FU, Hayer-Hartl M. Structure and function of RbcX, an assembly chaperone for hexadecameric Rubisco. Cell. 2007;129:1189-200.

7. Liu C, Young AL, Starling-Windhof A, Bracher A, Saschenbrecker S, Rao BV, Rao KV, Berninghausen O, Mielke T, Hartl FU, et al. Coupled chaperone action in folding and assembly of hexadecameric Rubisco. Nature 2010;463:197-202

8. Cai Z, Liu G, Zhang J, Li Y. Development of an activity-directed selection system enabled significant improvement of the carboxylation efficiency of Rubisco. Protein Cell. 2014:5:552-62.

9. Myat TL, Occhialini A, JohnAndralojc P, Martin AJP, Hanson MR. A faster Rubisco with potential to increase photosynthesis in crops. Nature. 2014:513:547-50
10. Lee H-M, Jeon B-Y, Oh M-K. Microbial production of ethanol from acetate by engineered Ralstonia eutropha. Biotechnol Bioprocess Eng. 2016;21:402-7.

11. Marc J, Grousseau E, Lombard E, Sinskey AJ, Gorret N, Guillouet SE. Over expression of GroESL in Cupriavidus necator for heterotrophic and autotrophic isopropanol production. Metab Eng. 2017:42:74-84.

12. Chen JS, Way JC, Dusel B, Torella JP. Production of fatty acids in Ralstonia eutropha $\mathrm{H} 16$ by engineering beta-oxidation and carbon storage. PeerJ. 2015;3:e1468.

13. Bi C, Su P, Müller J, Yeh YC, Chhabra SR, Beller HR, Singer SW, Hillson NJ. Development of a broad-host synthetic biology toolbox for Ralstonia eutropha and its application to engineering hydrocarbon biofuel production. Microb Cell Fact. 2013;12:1-10.

14. Schwartz E, Henne A, Cramm R, Eitinger T, Friedrich B, Gottschalk G. Complete nucleotide sequence of pHG1: a Ralstonia eutropha H16 megaplasmid encoding key enzymes of H2-based Lithoautotrophy and Anaerobiosis. J Mol Biol. 2003:332:369-83.

15. Pohlmann A, Fricke WF, Reinecke F, Kusian B, Liesegang H, Cramm R, Eitinge T, Ewering C, Pötter M, Schwartz E, et al. Genome sequence of the bioplastic-producing "Knallgas" bacterium Ralstonia eutropha H16. Nat Biotechnol. 2006:24:1257-62.

16. Cramm R. Genomic view of energy metabolism in Ralstonia eutropha H16. J Mol Microbiol Biotechnol. 2009;16:38-52

17. Kleihues L, Lenz O, Bernhard M, Buhrke T, Friedrich B. The $\mathrm{H} 2$ sensor of Ralstonia eutropha is a member of the subclass of regulatory [NiFe] hydrogenases. J Bacteriol. 2000;182:2716-24.

18. Kortlüke C, Horstmann K, Schwartz E, Rohde M, Binsack R, Friedrich B. A gene complex coding for the membrane-bound hydrogenase of Alcaligenes eutrophus H16. J Bacteriol. 1992;174:6277-89.

19. Bowien B, Kusian B. Genetics and control of CO 2 assimilation in the chemoautotroph Ralstonia eutropha. Arch Microbiol. 2002;178:85-93.

20. Burgdorf T, Lenz O, Buhrke T, van der Linden E, Jones AK, Albracht SPJ, Friedrich B. [NiFe]-Hydrogenases of Ralstonia eutropha H16: modula enzymes for oxygen-tolerant biological hydrogen oxidation. J Mol Microbiol Biotechnol. 2005;10:181-96.

21. Bernhard M, Benelli B, Hochkoeppler A, Zannoni D, Friedrich B. Functional and structural role of the cytochromeb subunit of the membraneboundhydrogenase complex of Alcaligenes eutrophus H16. Eur J Biochem. 1997:248:179-86

22. Tran-Betcke A, Warnecke U, Böcker C, Zaborosch C, Friedrich B. Cloning and nucleotide sequences of the genes for the subunits of NAD-reducing hydrogenase of Alcaligenes eutrophus H16. J Bacteriol. 1990;172:2920-9.

23. Li YJ, Wang MM, Chen YW, Wang M, Fan LH, Tan TW. Engineered yeast with a CO2-fixation pathway to improve the bio-ethanol production from xylosemixed sugars. Sci Rep. 2017;7:43875.

24. Zhao D, Yuan S, Xiong B, Sun H, Ye L, Li J, Zhang X, Bi C. Development of a fast and easy method for Escherichia coli genome editing with CRISPR/Cas9. Microb Cell Fact. 2016;15:205.

25. Xiong B, Li Z, Liu L, Zhao D, Zhang X, Bi C. Genome editing of Ralstonia eutropha using an electroporation-based CRISPR-Cas9 technique. Biotechnol Biofuels. 2018;11:172.

26. Reinecke F, Steinbüchel A. Ralstonia eutropha strain H16 as model organism for PHA metabolism and for biotechnological production of technically interesting biopolymers. J Mol Microbiol Biotechnol. 2009:16:91-108.

27. Parry Martin A J, John P. Andralojc: rubisco activity and regulation as targets for crop improvement. J Exp Bot. 2013:64:717-30.

28. Nathan J. Hillson RDRaJDK: j5 DNA assembly design automation software. ACS Synth Biol. 2012;1:14-21.

29. Quan J, Tian J. Circular polymerase extension cloning for high-throughput cloning of complex and combinatorial DNA libraries. Nat Protoc 2011;6:242-51.

30. Daniel G, Gibson LY. Ray-Yuan Chuang, J Craig Venter, Clyde A Hutchison III, Hamilton O Smith: enzymatic assembly of DNA molecules up to several hundred kilobases. Nat Methods. 2009;6:343-5.

31. Braunegg G, Lafferty RM. Rapid gas-chromatographic method for determination of poly-beta-hydroxybutyric acid in microbial Biomass. Appl Microbiol Biotechnol. 1978:6:29-3b7.

\section{Publisher's Note}

Springer Nature remains neutral with regard to jurisdictional claims in published maps and institutional affiliations. 